\title{
Fractional spinon excitations in the quantum Heisenberg antiferromagnetic chain
}

\author{
Martin Mourigal ${ }^{1,2,3 \star}$, Mechthild Enderle ${ }^{1}$, Axel Klöpperpieper ${ }^{4}$, Jean-Sébastien Caux ${ }^{5}$, \\ Anne Stunault ${ }^{1}$ and Henrik M. Rønnow ${ }^{2}$
}

\begin{abstract}
One of the simplest quantum many-body systems is the spin-1/2 Heisenberg antiferromagnetic chain, a linear array of interacting magnetic moments. Its exact ground state is a macroscopic singlet entangling all spins in the chain. Its elementary excitations, called spinons, are fractional spin-1/2 quasiparticles created and detected in pairs by neutron scattering. Theoretical predictions show that two-spinon states exhaust only $71 \%$ of the spectral weight and higher-order spinon states, yet to be experimentally located, are predicted to participate in the remaining. Here, by accurate absolute normalization of our inelastic neutron scattering data on a spin-1/2 Heisenberg antiferromagnetic chain compound, we account for the full spectral weight to within $99(8) \%$. Our data thus establish and quantify the existence of higher-order spinon states. The observation that, within error bars, the experimental line shape resembles a rescaled two-spinon one with similar boundaries allows us to develop a simple picture for understanding multi-spinon excitations.
\end{abstract}

$\bigcirc$ ne hundred years ago, Max von Laue and co-workers discovered X-ray diffraction ${ }^{1}$, thereby giving birth to the field of crystallography to which we owe much of our understanding of materials on the atomic scale. The very first diffraction image was recorded from a single crystal of copper sulphate pentahydrate ${ }^{1,2}$. In addition to vast practical use including herbicide, wood impregnation and algae control in swimming pools, copper sulphate also carries great educational importance. Generations of school children have been inspired in chemistry classes across the globe by growing from evaporating solution beautiful blue crystals of copper sulphate (in 2008, artist Roger Hiorns created an installation called Seizure ${ }^{3}$ covering an entire apartment in copper sulphate crystals). When cooled close to absolute zero temperature, copper sulphate has even more fascinating lessons to teach-it becomes a quantum spin liquid. Moreover, it materializes one of the simplest models hosting complex quantum many-body physics, the one-dimensional spin-1/2 Heisenberg antiferromagnet, for which there exists an exact analytic solution-namely the Bethe ansatz ${ }^{4}$.

Quantum spin liquid ground states entangle a macroscopic number of spins and give rise to astonishing and counterintuitive phenomena. Quantum spin liquids occur in a variety of contexts ranging from the quantum spin Hall effect ${ }^{5,6}$ and high$T_{\mathrm{c}}$ superconductivity $^{7-9}$ to confined ultracold gases and carbon nanotubes ${ }^{10}$. A particularly clear form of a gapless algebraic quantum spin liquid is realized in a one-dimensional array of spins-1/2 that are coupled by nearest-neighbour isotropic exchange, the spin-1/2 Heisenberg antiferromagnetic chain. At zero temperature, this spin liquid is critical with respect to long-range antiferromagnetic order as well as with respect to dimerization ${ }^{11,12}$. Its emerging gapless fractionalized excitations are called spinons ${ }^{13}$. The concept of fractional excitations has been applied to magnetic monopoles in spin ice ${ }^{14-17}$, kagome and hyper-kagome lattices ${ }^{18}$, the quantum Hall effect ${ }^{19-22}$, conducting polymers ${ }^{23,24}$, and even to certain spin arrays with local spin larger than $1 / 2$ (refs 25, 26). For the prototypical spin-1/2 Heisenberg antiferromagnetic chain, exact calculations of the dynamic structure factor over the whole range of the spectrum have become available. They reveal that two-spinon states exhaust $71 \%$ of the first frequency moment sum rule ${ }^{27}$. Including four-spinon states brings that level to $98(1) \%$ (ref. 28). The qualitative characteristics of two-spinon excitations, a continuum-like spectrum with linearly dispersing low-energy onset, are evidenced by inelastic neutron scattering on numerous compounds ${ }^{29-43}$. Among them, there are various quantitative attempts of an absolute comparison to theory ${ }^{30,37,41}$. However, none was sufficiently accurate to distinguish between an excitation continuum made of only two-spinon states and that composed of two- and higher-order spinon states, bearing a $~ 30 \%$ larger spectral weight. The main sources of uncertainty come from the need to normalize the neutron scattering intensity to that of a reference Vanadium standard and from the role of other intrinsic and extrinsic sources of bias such as covalency, self-absorption, atomic zero point and thermal motion, and the limits of the dipole approximation used in the interpretation of the neutron crosssection. The recent most accurate study, on the cuprate compound $\mathrm{Sr}_{2} \mathrm{CuO}_{3}$, finds only $80 \%$ of the predicted spectral intensity ${ }^{41}$.

Here we present a totally different approach to quantifying the full correlator, including two- and higher-spinon contributions. A magnetic field $\mathbf{H} \| \mathbf{z}$ large compared with the antiferromagnetic exchange aligns all spins parallel. This fully polarized state, $\left\langle S_{n}^{z}\right\rangle=S$, is an eigenstate of the Heisenberg Hamiltonian $\mathcal{H}=J \sum_{n} \mathbf{S}_{n} \mathbf{S}_{n+1}-g \mu_{\mathrm{B}} H S^{z}$, and identical to the classical ground state, which we obtain if we neglect all commutation relations of spin operators. Consequently, dispersion and intensity of the low-energy excitation spectrum are correctly described by linear spin-wave theory for which the elementary quasiparticles are non-interacting magnons. The classical magnon dispersion in

${ }^{1}$ Institut Laue-Langevin, BP 156, 38042 Grenoble Cedex 9, France, ${ }^{2}$ Laboratory for Quantum Magnetism, École Polytechnique Fédérale de Lausanne (EPFL), 1015 Lausanne, Switzerland, ${ }^{3}$ Institute for Quantum Matter and Department of Physics and Astronomy, Johns Hopkins University, Baltimore, Maryland 21218, USA, ${ }^{4}$ Technische Physik, Universität des Saarlandes, D-66041 Saarbrücken, Germany, ${ }^{5}$ Institute for Theoretical Physics, University of Amsterdam, Science Park 904, 1090 GL Amsterdam, The Netherlands. *e-mail: mourigal@pha.jhu.edu 


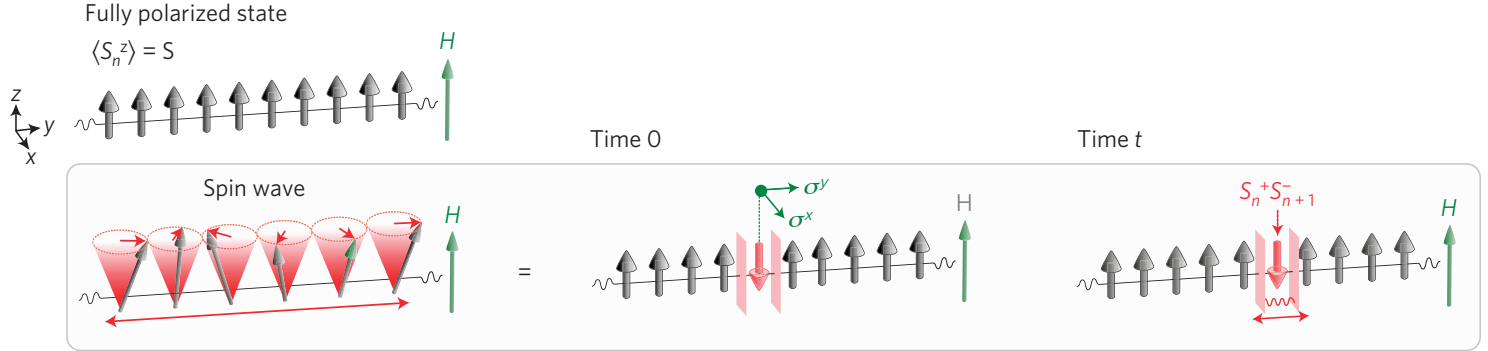

b

Zero magnetic field state
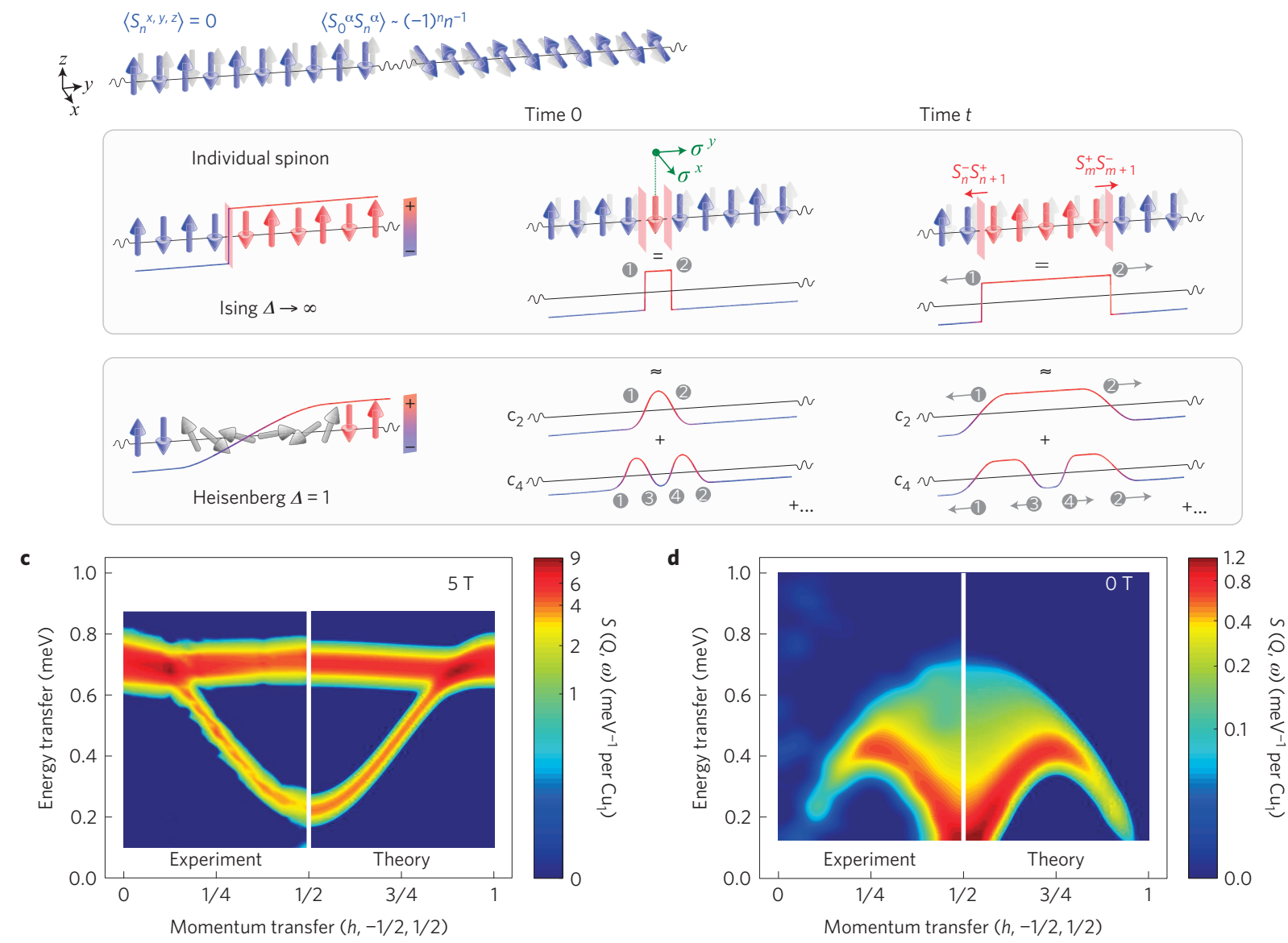

Figure 1 | Schematic representation of the magnetic excitations in a spin-1/2 (Heisenberg) antiferromagnetic chain and overview of the neutron scattering results for $\mathrm{CuSO}_{4} \cdot \mathbf{5 D}_{2} \mathbf{O}$. a, Fully polarized (saturated) state. The creation of a magnon by inelastic scattering of a neutron can be imagined as a single spin flip. The Zeeman energy prevents any growth of the flipped section that propagates like a single entity. This magnon can classically be visualized as a spin wave, a coherent precession of the local spin expectation value around the field direction. $\mathbf{b}$, Zero magnetic field state. Snapshots of large antiferromagnetically correlated regions of the ground state. The spins could be found in a locally antiferromagnetic configuration with equal probability in any direction (for example, the opposite one (shadows)). The neutron acts on the singlet ground state and excites triplet states, which we may imagine as a local spin flip surrounded by two domain walls, and which individually correspond to a spinon carrying spin- $1 / 2$. The spatial extent of a spinon depends on the anisotropy: in the Ising limit, a local spin flip decomposes into two spinons; in the Heisenberg limit, it decomposes into a rapidly converging series of states containing two, four and higher even numbers of such spinons. c, Intensity maps of the experimental and theoretical magnon dispersion in the fully polarized phase of $\mathrm{CuSO}_{4} \cdot 5 \mathrm{D}_{2} \mathrm{O}$ for $\mu_{0} \mathrm{H}=5 \mathrm{~T}>\mu_{0} \mathrm{H}_{\text {sat }}$ and $T \sim 100 \mathrm{mK}$, above the Néel transition temperature to three-dimensional antiferromagnetic ordering. The two observed branches (flat and cosine-shaped) are associated with two non-equivalent $\mathrm{Cu}^{2+}$ sites in $\mathrm{CuSO}_{4} \cdot 5 \mathrm{D}_{2} \mathrm{O}\left(\mathrm{Cu}_{1}\right.$ and $\mathrm{Cu}_{2}$, respectively). The cosine-shaped dispersion corresponds to the excited magnon of the saturated Heisenberg antiferromagnetic $\mathrm{Cu}_{1} \mathrm{chain}$ and the flat branch around $0.7 \mathrm{meV}$ is a transition between two local Zeeman levels of the decoupled $\mathrm{Cu}_{2}$ sites. $\mathbf{d}$, Intensity colour maps of the experimental inelastic neutron scattering spectrum of the $\mathrm{Cu}_{1}$ chain spins in the zero-field phase of $\mathrm{CuSO}_{4} \cdot 5 \mathrm{D}_{2} \mathrm{O}$, and theoretical two- and four-spinon dynamic structure factor.

the fully polarized phase has already been successfully employed to determine the microscopic parameters of the Hamiltonian of a two-dimensional frustrated quantum antiferromagnet ${ }^{44}$. Here, we go one step further and determine not only the microscopic parameters from the dispersion of the magnon, but also exploit its wave-vector-independent intensity to obtain an absolute intensity scale. Having fixed energy and intensity scale at high magnetic field, in the fully polarized classical phase, the quantum theory is tested against the zero-field data without any adjustable parameters. This approach avoids numerous uncertainties of previous attempts 


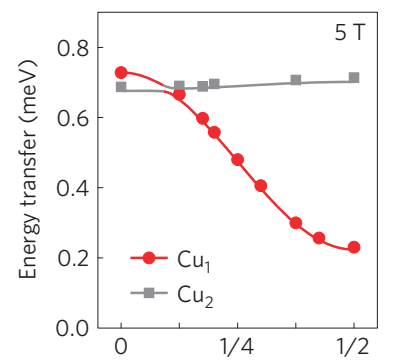

Momentum transfer $(h,-1 / 2,-1 / 2)$

d

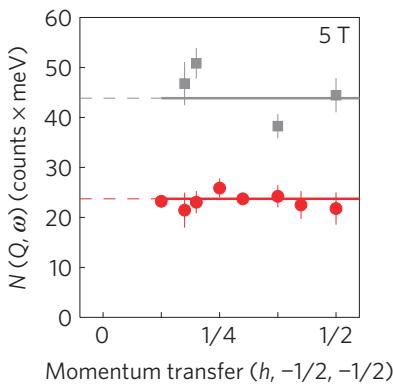

f

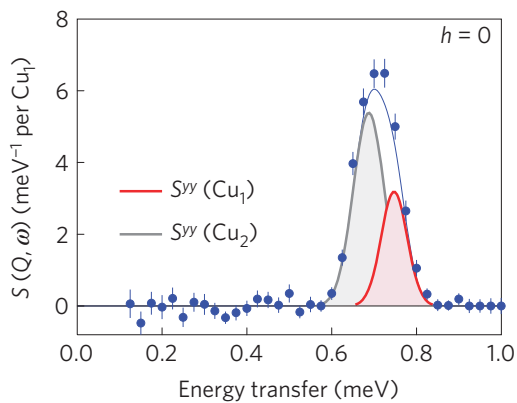

h

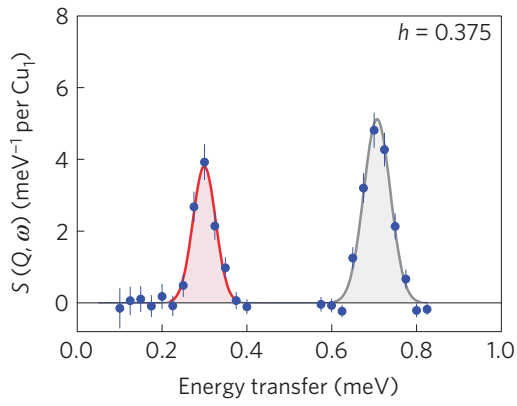

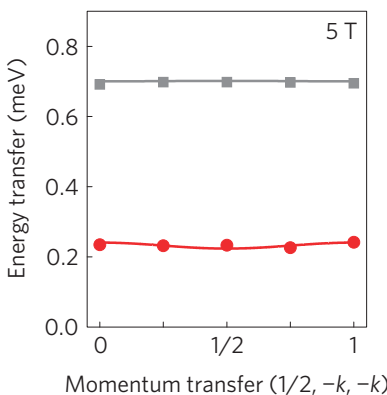

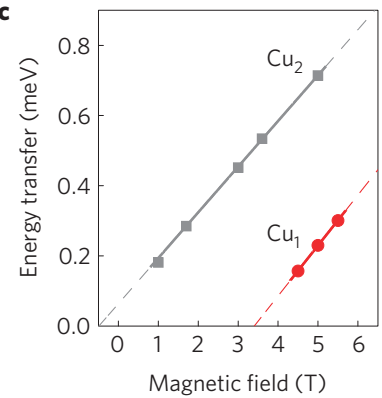

e

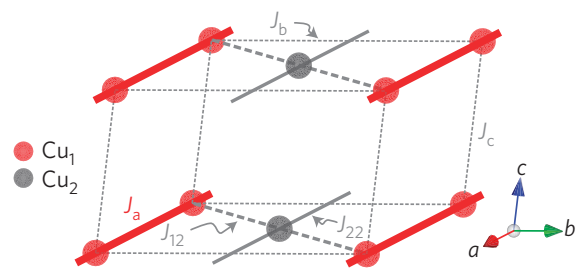

g

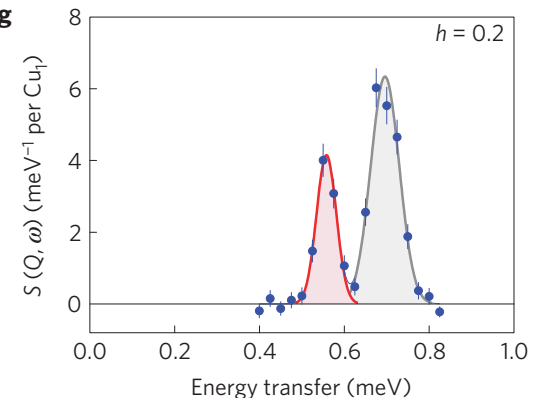

i

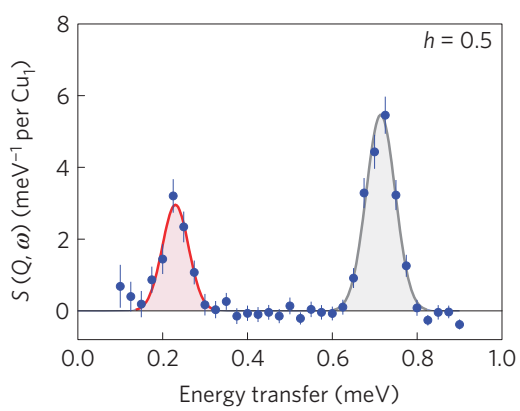

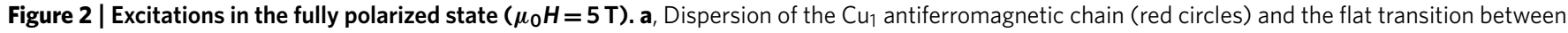
local Zeeman states of $\mathrm{Cu}_{2}$ (grey squares). The lines show the spin-wave fit described in the Supplementary Information. $\mathbf{b}$, Flat dispersions along ( $0, k, k$ ) evidence that $\mathrm{Cu}_{1}$ and $\mathrm{Cu}_{2}$ spins are decoupled in this direction. The lines represent the spin-wave fit. $\mathbf{c}$, The Zeeman shift of $\mathrm{Cu}_{1}$ and $\mathrm{Cu} \mathrm{u}_{2}$ branches at the

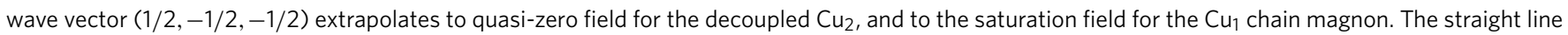
fits correspond to $g_{1}$ and $g_{2}$ given in the text. $\mathbf{d}$, Intensity of the $\mathrm{Cu}_{1}$ collective antiferromagnetic chain excitation and the flat transition between local Zeeman states of $\mathrm{Cu}_{2}$. e, Magnetic primitive cell, triclinic symmetry. Chain-forming $\mathrm{Cu}_{1}$ sites (red) at [0,0,0] and decoupled Cu sites (grey) at $[1 / 2,1 / 2,0] . \mathbf{f}-\mathbf{i}$, Selected energy scans at constant wave vector $(h,-1 / 2,-1 / 2)$ together with the spin-wave fit, described in the Supplementary Information. Error bars correspond to 1 s.d.

and allows one to verify the role of higher-order spinon states quantitatively.

In Fig. 1, we illustrate schematically the characteristic differences of the ground state and the excitations in the zero-field and the fully polarized phase. The magnon in the fully polarized state can be understood as a firmly bound domain wall pair that propagates and delocalizes as a single entity (Fig. 1a). This results in a discrete energy-momentum dispersion relation of the magnon, evidenced in the intensity colour plots (Fig. 1c). Domain wall propagation is achieved through the terms $S_{n}^{\alpha} S_{n+1}^{\alpha}$ in $\mathcal{H}$ with $\alpha=x, y$. The excitation amplitude (the magnon eigenvector) is therefore always transverse to the applied magnetic field $\mathbf{H} \| \mathbf{z}$.

In zero magnetic field the spins-1/2 entangle into a macroscopic singlet $\boldsymbol{S}_{\text {tot }}=\sum_{n} \mathbf{S}_{n}=0$, where local spin projections are no longer good quantum numbers, $\left\langle S_{n}^{\alpha}\right\rangle=0, \alpha=x, y, z$. Nevertheless, at $T=0$ the two-spin correlations decay only algebraically, $\left\langle S_{0}^{\alpha} S_{n}^{\alpha}\right\rangle \propto(-1)^{n} n^{-1}$, indicating infinitely large correlated antiferromagnetic regions. Snapshots of such a correlated region are shown in Fig. 1b. Within such a region, the inelastically scattered neutron provokes $S_{\text {tot }}=1$ excitations, which we may first imagine as a 


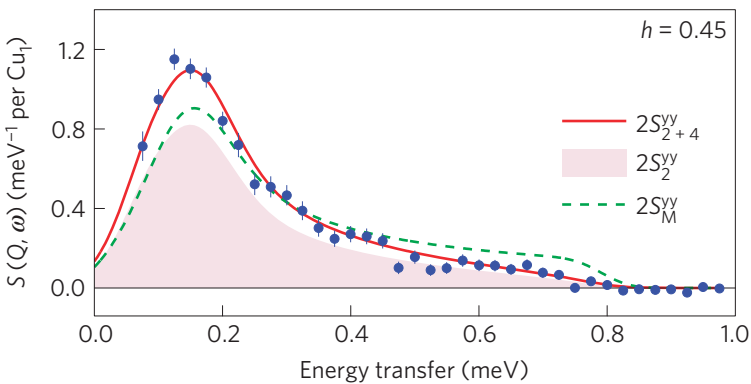

b

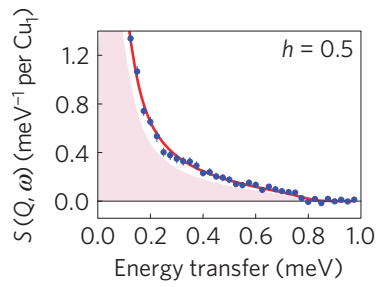

d
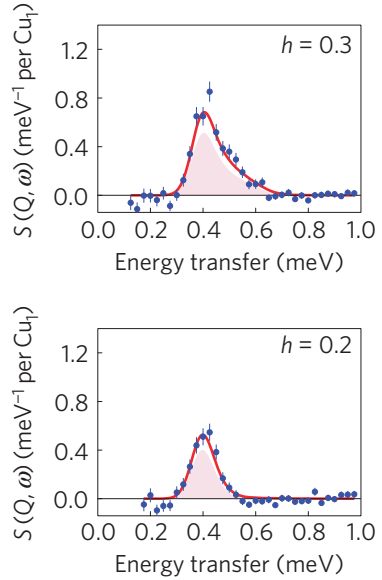

c
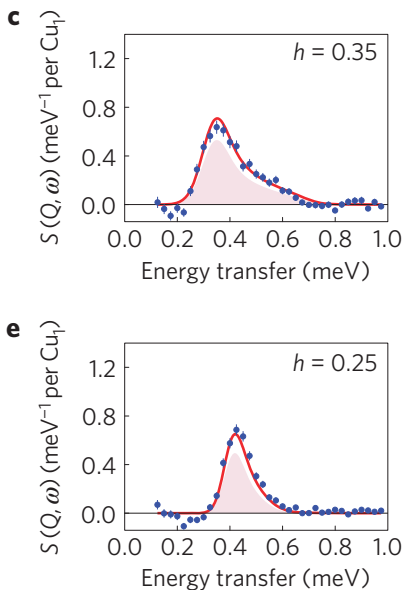

g

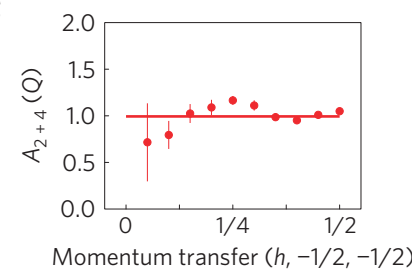

Figure 3 | Excitations in zero magnetic field. a-f, Experimentally determined $S(h, \omega)=S^{y y}(h, \omega)+S^{z z}(h, \omega)$ (blue circles) in comparison to the two- and four-spinon structure factor $2 S_{2+4}^{y y}(h, \omega)$ (red line), and the two-spinon-only intensity $2 S_{2}^{y y}(h, \omega)$ (rose shaded area), for typical wave vectors $\mathbf{Q}=(h,-1 / 2,-1 / 2)$ with $h$ as indicated in the legend. The theoretical structure factors are shown for $J_{a}=0.256 \mathrm{meV}$, obtained from a global fit. In $\mathbf{a}$, the experimental data are compared not only to the exact two- and four-spinon structure factor (red line) but also to a fit to the Müller ansatz ${ }^{46} 2 A_{M} S_{M}^{y y}(h, \omega)$ (green dashed line) with free amplitude $A_{M}$, see text and Supplementary Information. $\mathbf{g}$, Fitted amplitude $A_{2+4}(h)$ for $2 S_{2+4}^{y y}(h, \omega)$, with $J_{a}=0.252 \mathrm{meV}$ fixed as determined from the spin wave fit in the fully polarized phase (red circles). The red line corresponds to $A_{2+4}=0.99$ obtained from the same global fit as for the other panels. Error bars correspond to 1 s.d.

local spin flip surrounded by two domain walls. These domain walls delocalize owing to the terms of the Hamiltonian that are transverse to the quantization axis, and propagate individually and independently, in contrast to the fully polarized state where they propagate in pairs as a firmly bound entity.

The elementary excitation, the spinon, carries spin-1/2 and can be pictorially associated with an individually propagating domain wall. Spinons separate two sections of the macroscopic singlet ground state wavefunction that are phase-shifted by $\pi$. They are easy to visualize (Fig. 1b) in the extreme Ising limit $(\Delta \rightarrow \infty$ in $\mathbf{S}_{n} \mathbf{S}_{n+1}=S_{n}^{x} S_{n+1}^{x}+S_{n}^{y} S_{n+1}^{y}+\Delta S_{n}^{z} S_{n+1}^{z}$ ), where they represent an abrupt domain wall between the two distinct antiferromagnetic orders. Approaching the Heisenberg limit, $\Delta=1$, the local spin flip can no longer be represented by two spinons alone, but rather

decomposes in a rapidly converging series of states containing two, four and higher even numbers of spinons.

Whereas each spinon has a discrete energy-momentum relation, the excitation spectrum is composed of spinon pairs (and higher even-numbered spinon states with $S_{\text {tot }}=1$ ), and will therefore be continuous. This characteristic continuous spectrum is indeed observed at zero field, both experimentally and in exact calculations of the two- and four-spinon contributions (Fig. 1d). In contrast to magnons, the spinon excitation amplitude is identical for all three orthogonal directions, $\alpha=x, y, z$. Precise calculations for the two- and four-spinon spectra (which represent around 98(1)\% of the full response function in the thermodynamic limit) have become available $e^{28}$, and we demonstrate in the following that our experimental results confirm the abstract spinon concept accurately on the quantitative level.

We performed inelastic neutron scattering experiments on large single crystals of $\mathrm{CuSO}_{4} \cdot 5 \mathrm{D}_{2} \mathrm{O}$, cooled in a dilution refrigerator to $\sim 100 \mathrm{mK}$ just above the transition temperature to a threedimensional antiferromagnetic Néel state. There are two $\mathrm{Cu}^{2+}$ sites in the elementary unit cell, $\mathrm{Cu}_{1}$ at $[0,0,0]$, and $\mathrm{Cu}_{2}$ at $[1 / 2,1 / 2,0]$, which each provide a localized spin-1/2 (Fig. 2e). All antiferromagnetic exchange is overcome by the Zeeman energy in a modest magnetic field of $5 \mathrm{~T}$. In this fully polarized phase, we observe discrete excitations, well described by a resolution-convoluted $\delta$-function, the signature of a magnon (Fig. 1c). We observe a dispersive branch with $0.517(9) \mathrm{meV}$ bandwidth along $\mathbf{a}^{*}$ and a minimum at $h=1 / 2$ (Fig. 2a). This dispersive branch reflects the principal antiferromagnetic exchange interaction between neighbouring $\mathrm{Cu}_{1}$ spins, which hence form chains along a. Along $(0, k, k)$, perpendicular to the chain direction, the bandwidth is smaller than the experimental error of $0.007 \mathrm{meV}$ (Fig. 2b) and evidences negligible coupling between individual spin chains. A second branch at energy $2 g_{2} \mu_{\mathrm{B}} H S^{z}$ (grey in Fig. 2) is flat both along $(h, 0,0)$ (bandwidth $0.048(6) \mathrm{meV}$ ) and $(0, k, k)$ (bandwidth $<0.007 \mathrm{meV}$ ). As a function of magnetic field, the dispersive branch emerges from zero energy at the saturation field strength, whereas the energy of the flat branch is directly proportional to the magnetic field (Fig. 2c). The field dependence and vanishing bandwidth of the flat branch reveal an essentially decoupled spin site $\left(\mathrm{Cu}_{2}\right)$, where the neutron excites the local spin from the lower to the upper Zeeman level. The Zeeman shifts of the dispersive and of the flat branch are slightly different, as expected for the Landé $g$-factors $g_{1}$ and $g_{2}$ of two different crystallographic sites. This scenario is also confirmed by our spin-wave calculations, which take into account various potential exchange paths sketched in Fig. 2e (Methods), and provide the dominant exchange between the $\mathrm{Cu}_{1}$ spins as $J_{\mathrm{a}}=0.252(17) \mathrm{meV}$. The interchain interactions are found to be so small that a one-dimensional treatment for the $\mathrm{Cu}_{1}$ spins remains fully applicable for temperatures above the three-dimensional Néel ordering.

At zero magnetic field, the Zeeman levels of the decoupled $\mathrm{Cu}_{2}$ spins are not split, the flat branch has energy zero, and only the chain-forming $\mathrm{Cu}_{1}$ spins contribute to the inelastic spectrum. We observe a sine-shaped lower boundary of the continuous scattering, with maximum onset-energy $0.402(2) \mathrm{meV}$ at $h=1 / 4$. This experimentally determined spinon bandwidth agrees within $2 \%$ error with the theoretical prediction $\pi / 2 J_{\mathrm{a}}=0.406(7) \mathrm{meV}$, with $J_{\mathrm{a}}$ determined from the bandwidth in the fully polarized phase. We thus confirm experimentally that the energy of spinon excitations is quantum renormalized upwards by a factor $\frac{\pi}{2}$ compared with classical magnons ${ }^{45}$.

To compare the observed intensities to the theoretical two- and four-spinon dynamic structure factor, we consider the inelastic neutron cross-section

$$
\frac{\mathrm{d}^{2} \sigma}{\mathrm{d} \Omega \mathrm{d} \omega}=\frac{k_{\mathrm{f}}}{k_{\mathrm{i}}}\left(\gamma r_{0}\right)^{2} \sum_{\alpha=x, y, z}\left(g_{1} f(\mathbf{Q})\right)^{2}\left(1-\hat{Q}^{\alpha} \hat{Q}^{\alpha}\right) S^{\alpha \alpha}(\mathbf{Q}, \omega)
$$




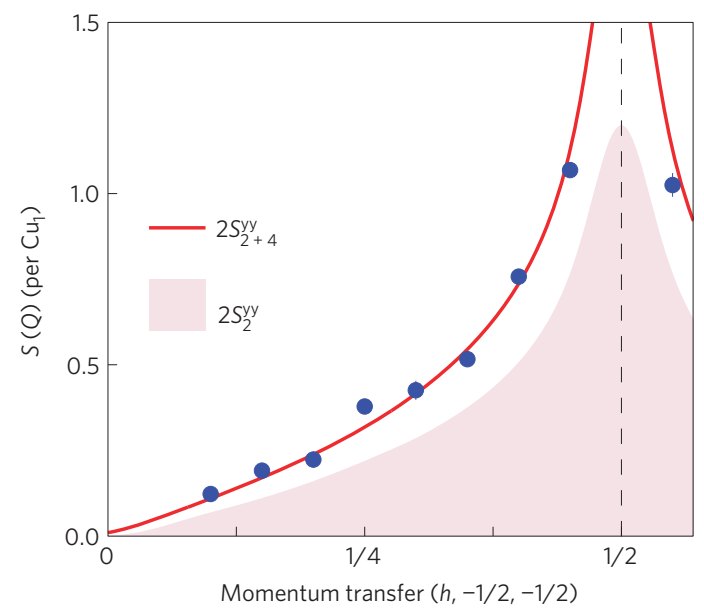

b

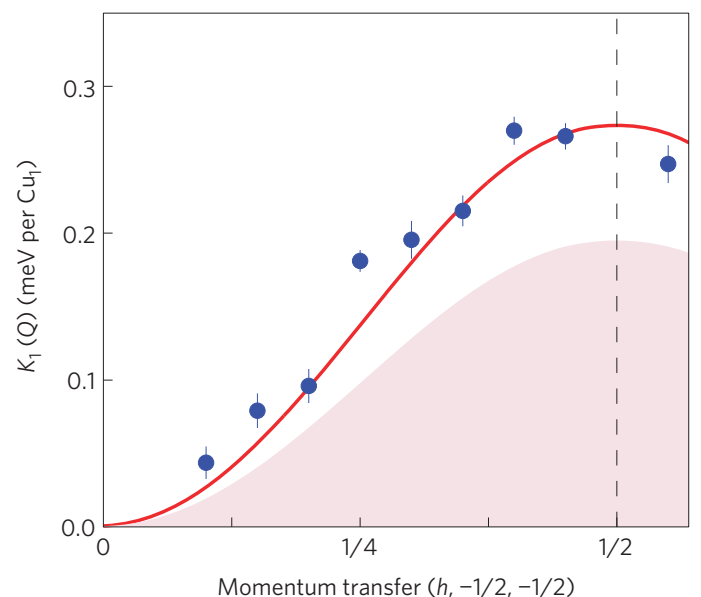

Figure 4 | Sum rules. a,b, Experimental structure factor

$S(\mathbf{Q})=\int S(\mathbf{Q}, \omega) \mathrm{d} \omega=\int\left(S^{y y}(\mathbf{Q}, \omega)+S^{z z}(\mathbf{Q}, \omega)\right) \mathrm{d} \omega$ (blue circles; a) and first frequency moment $K_{1}(\mathbf{Q})=\int \omega S(\mathbf{Q}, \omega) \mathrm{d} \omega$ (blue circles; $\mathbf{b}$ ) as a function of momentum transfer $\mathbf{Q}=(h,-1 / 2,-1 / 2)$ versus two- and four-spinon calculations, $2 S_{2+4}^{y y}(\mathbf{Q}, \omega)$ (red line) and $2 S_{2}^{y y}(\mathbf{Q}, \omega)$ (shaded area). Error bars correspond to 1 s.d.

Here, $k_{\mathrm{i}}, k_{\mathrm{f}}$ denote the variable incoming and fixed outgoing neutron wave vector, $\gamma$ is the gyromagnetic ratio of the neutron, $r_{0}$ is the electron Bohr radius, $f(\mathbf{Q})$ is the form factor of the electronic shell responsible for the $\mathrm{Cu}_{1}$ spin, taken at the total momentum transfer $\mathbf{Q}$, and $S^{\alpha \alpha}(\mathbf{Q}, \omega)=1 /(2 \pi)^{4} \hbar \iint\left\langle S^{\alpha}(0,0) S^{\alpha}(\mathbf{r}, t)\right\rangle \exp (i(\mathbf{Q}$. $\mathbf{r}-\omega t)) \mathrm{d}^{3} \mathbf{r} \mathrm{d} t$ is the dynamic structure factor, where $\alpha=x, y, z$ denote the directions $\mathbf{x} \| \mathbf{Q}, \mathbf{y} \perp \mathbf{Q}$ in the scattering plane, and $\mathbf{z}$ perpendicular to the scattering plane.

In our experiment, the magnetic field $\mathbf{H} \| \mathbf{z}=[0,-1,1]$ is perpendicular to the scattering plane. The theoretical magnon intensity in the fully polarized phase is given by $S^{x x}(\mathbf{Q}, \omega)=S^{y y}(\mathbf{Q}, \omega)=(S / 2) \delta(\omega-\omega(h))$ per chain spin $\mathrm{Cu}_{1}$. Of these, only $S^{y y}(\mathbf{Q}, \omega)$ is visible, because the neutron scatters exclusively from magnon eigenvectors perpendicular to the total momentum transfer $\mathbf{Q} \| \mathbf{x}$ as expressed by the factor $\left(1-\hat{Q}^{\alpha} \hat{Q}^{\alpha}\right)$ in the cross-section. We therefore identify the observed $k_{\mathrm{i}}$-normalized intensity (Supplementary Information) with $N(\mathbf{Q}, \omega) S^{y y}(\mathbf{Q}, \omega)=$ $N(\mathbf{Q}, \omega) S / 2$. We observe in the experiment that $N(\mathbf{Q}, \omega)$ does not depend on $\mathbf{Q}$ or $\omega$ (Fig. 2d). $N(\mathbf{Q}, \omega)=\bar{N}$ therefore contains all prefactors in the neutron scattering cross-section, including orbital and covalency effects.

The zero-field data are then normalized by the factor $\bar{N}$ obtained from the fully saturated phase, and thus can be directly compared to the dynamic structure factor per $\mathrm{Cu}_{1}$ spin. For an isotropic ground state we have $S^{x x}(\mathbf{Q}, \omega)=S^{y y}(\mathbf{Q}, \omega)=S^{z z}(\mathbf{Q}, \omega)$, but only $S^{y y}(\mathbf{Q}, \omega)+S^{z z}(\mathbf{Q}, \omega)=2 S^{y y}(\mathbf{Q}, \omega)$ contribute to the inelastic scattering cross-section.

In Fig. 3 we compare the normalized zero-field inelastic spectra to theoretical predictions. Before exact calculations were available, experimental data were usually compared to the Müller ansatz ${ }^{46}$, an approximation of the two-spinon continuum with an artificial cutoff at the upper two-spinon boundary. Evidently, the line shape of the Müller ansatz does not very well describe our experimental data in that it underestimates the low-energy part of the spectrum and overestimates the high-energy part (Fig. 3a). Next, we compare our data to the exact two- and four-spinon dynamic structure factor $2 S_{2+4}^{y y}(\mathbf{Q}, \omega)$ (ref. 28). For this comparison we introduce a $\mathbf{Q}$-dependent prefactor, $A_{2+4}(\mathbf{Q})$, which equals 1 if the theory describes the normalized inelastic intensities perfectly. With the exchange $J_{\mathrm{a}}=0.252 \mathrm{meV}$ fixed to the value determined by the spin-wave fit to all data of the fully polarized phase (Supplementary Information) we fit for each wave vector $\mathbf{Q}=(h,-1 / 2,-1 / 2)$ the prefactor $A_{2+4}(h)$ of the two- and four-spinon structure factor $2 S_{2+4}^{y y}(\mathbf{Q}, \omega)$. We obtain prefactors close to 1 and essentially independent of $\mathbf{Q}$, with $\overline{A_{2+4}(h)}=1.03(9)$ (Fig. 3g). A simultaneous fit of all zero-field data with free $J_{\mathrm{a}}$ yields $J_{\mathrm{a}}=0.256(1) \mathrm{meV}$ and the global prefactor $A_{2+4}=0.99(8)$. This fit is shown as red lines in Fig. 3, and is indistinguishable from lines with $A_{2+4}=1$. To illustrate the importance of the four-spinon contribution, Fig. $3 \mathrm{a}-\mathrm{f}$ also shows the exact two-spinon-only structure factor as a shaded area. Inside the two-spinon boundaries, the two- and four-spinon continua have a similar line shape. Therefore, fits to the two-spinon-only structure factor could approximately model the data, but would require an increase of the prefactor to 1.4(1). Owing to our accurate absolute normalization of the neutron data we can therefore establish that two-spinon states account for only $74(6) \%$ of the measured spectral weight. We thereby unambiguously demonstrate that higher spinon states contribute significantly with $26(6) \%$ to the spectrum.

The essential properties of four-spinon excitations can be captured using the pictures of Fig. 1b. In the Ising limit $(\Delta \rightarrow \infty)$, a localized spin flip exactly projects onto a state with two spinons. As these domain walls are localized, the state immediately after the spin flip can be represented as a combination of two-spinon states with a broad momentum distribution. In the Heisenberg case $(\Delta \rightarrow 1)$ however, because each spinon is an extended object, the initial $(t=0)$ state with a local spin flip must be decomposed into a quantum mechanical superposition of (mostly) two- and fourspinon states weighted by spinon-momentum-dependent complex amplitudes. The evolution of that state (at a different position and later time $t>0$ ) is encoded in the spin-spin correlator such that two-spinon excitations contribute if one spinon propagates at the appropriate velocity. The leading four-spinon parts of the correlator closely follow that of the two-spinon states; they resemble two-spinon contributions but with two additional spinons added with close to zero momentum and energy. As a consequence, the four-spinon correlation weight is almost entirely contained within the boundaries of the two-spinon continuum, where it approximatively follows the same line shape. In the Supplementary Information we complement this description of two-spinon and four-spinon states on the basis of the Bethe ansatz.

Figure 4, which illustrates the static structure factor $S(\mathbf{Q})$ and the first frequency moment $\int \omega S(\mathbf{Q}, \omega) \mathrm{d} \omega$, additionally confirms that two- and four-spinon excitations together essentially exhaust the spectral weight and the first moment sum rule. The two-spinononly contribution is again shown as a shaded area, and can clearly not account for the observed intensity. Our work thus proves quantitatively the validity of the spinon concept for the excitation spectrum of the spin-1/2 Heisenberg chain.

We exploit in this work that a large magnetic field quenches $\mathrm{CuSO}_{4} \cdot 5 \mathrm{D}_{2} \mathrm{O}$ from a macroscopically entangled quantum state 
into the fully polarized state that can be described classically. Domain wall pair excitations have then a finite threshold energy and are bound or confined by the magnetic field. The resulting quasiparticle, the magnon, is correctly described by the classical spin-wave theory. We use its known energy and intensity to quantitatively confirm the abstract concept of fractional spinon excitations out of the macroscopically entangled quantum state at zero field in a real material. The theoretical spinon concept is fully confirmed by our experiment-the spinon has a $\pi / 2$ larger bandwidth than the magnon, the line shape of the energy scans corresponds to the exact two- and four-spinon dynamic structure factor, and both two- and four-spinon contributions are needed to account for the experimentally observed intensity, spectral weight, static structure factor and first moment sum rule.

\section{Methods}

Full methods are available in the Supplementary Information.

Inelastic neutron scattering. Inelastic neutron scattering experiments were performed on the cold triple-axis spectrometer IN14 at the Institut Laue-Langevin (Grenoble), equipped with a PG(002)-monochromator and analyser and final neutron wave vector fixed at $k_{\mathrm{f}}=1.15 \AA^{-1}$ (full-width at half-maximum energy resolution of $0.078 \mathrm{meV}$ ) or $1.025 \AA^{-1}$ (full-width at half-maximum energy resolution of $0.05 \mathrm{meV}$ ). The sample was a $2 \mathrm{~cm}^{3}$ single crystal of deuterated $\mathrm{CuSO}_{4} \cdot 5 \mathrm{D}_{2} \mathrm{O}$ oriented with $(h, 0,0)$ and $(0, k, k)$ in the horizontal scattering plane, and cooled to $100 \mathrm{mK}$ by a dilution insert inside either an ILL orange-type cryostat or a $5.5 \mathrm{~T}$ vertical magnet. The temperature was chosen just above the Néel temperature ${ }^{47}$ and the absence of three-dimensional antiferromagnetic ordering was confirmed by the vanishing scattering intensity at the magnetic Bragg reflection. The non-magnetic background from incoherent elastic scattering was derived from the high-field measurements and subtracted from all spectra.

Spin-wave theory. The spectrum from the high-field fully polarized state was analysed by a linear spin-wave theory fit to yield exchange parameters, $g$-factors and absolute intensity. We obtained a dominant exchange between nearest-neighbour $\mathrm{Cu}_{1}$ spins, $J_{\mathrm{a}}=0.252(17) \mathrm{meV}$. Other magnetic interactions in the material were found to be small or negligible, $J_{b}+J_{c}<0.004(7) \mathrm{meV}, J_{22}=-0.012(18) \mathrm{meV}$ and $J_{12}=-0.020(22) \mathrm{meV}$. The $g$-factors for the magnetic field along $[0,1,-1]$ were determined for each copper site from the Zeeman shift of their respective branch. The theoretical magnon intensity was obtained in the fully polarized phase as $S^{x x}(\mathbf{q}, \omega)=S^{y y}(\mathbf{q}, \omega)=(S / 2) \delta(\omega-\omega(h))$ per $\mathrm{Cu}_{1}$.

Zero-field model. The zero-field data were analysed by comparing to calculations including two- and four-spinon states. Their exact contributions to the zero-temperature dynamical structure factor of the spin-1/2 Heisenberg isotropic chain were obtained directly in the thermodynamic limit using the vertex operator approach $^{48}$ based on the exact solvability of the Heisenberg model ${ }^{28}$. This theoretical dynamic structure factor was compared to the zero-field data after convolution to a normalized two-dimensional Gaussian profile to account for the finite experimental energy and momentum resolution in the experiment. From a global least-squares fit to the entire data set we obtained $J_{\mathrm{a}}=0.256(1) \mathrm{meV}$, a global amplitude $A_{2+4}=0.993(84)$, and an energy resolution of $0.078 \mathrm{meV}$, in perfect agreement with the measured resolution at zero energy transfer.

Received 14 March 2013; accepted 13 May 2013; published online 16 June 2013

\section{References}

1. Friedrich, W., Knipping, P. \& Laue, M. Interferenz-Erscheinungen bei Röntgenstrahlen. Proc. Bavarian Acad. Sci. (Sber. Bayer. Akad. Wiss.) 303-322 (1912).

2. Friedrich, W., Knipping, P \& Laue, M. Interferenz-Erscheinungen bei Röntgenstrahlen. Naturwissenschaften 39, 361-369 (1952).

3. Morton, T., Charlesworth, J. J. \& Lingwood, J. Roger Hiorns: Seizure (Artangel, 2008).

4. Bethe, H. Zur Theorie der Metalle. I. Eigenwerte und Eigenfunktionen der linearen Atomkette. Z. Phys. A 71, 205-226 (1931).

5. Raghu, S., Qi, X-L., Honerkamp, C. \& Zhang, S-C. Topological Mott insulators. Phys. Rev. Lett. 100, 156401 (2008).

6. Kane, C. L. \& Mele, E. J. Quantum spin Hall effect in graphene. Phys. Rev. Lett. 95, 226801 (2005).

7. Anderson, P. W. The resonating valence bond state in $\mathrm{La}_{2} \mathrm{CuO}_{4}$ and superconductivity. Science 235, 1196-1198 (1987).

8. Kivelson, S. A., Rokhsar, D. S. \& Sethna, J. P. Topology of the resonating valence-bond state: Solitons and high- $T_{c}$ superconductivity. Phys. Rev. B 35, 8865-8868 (1987).
9. Lee, P. A. From high temperature superconductivity to quantum spin liquid: Progress in strong correlation physics. Rep. Prog. Phys. 71, 012501 (2008).

10. Meng, Z. Y. et al. Quantum spin liquid emerging in two-dimensional correlated Dirac fermions. Nature 464, 847-851 (2010).

11. Lieb, E., Schultz, T. \& Mattis, D. Two soluble models of an antiferromagnetic chain. Ann. Phys. 16, 407-466 (1961).

12. Affleck, I. Quantum spin chains and the Haldane gap. J. Phys. Condens. Matter 1, 3047-3072 (1989).

13. Faddeev, L. D. \& Takhtajan, L. A. What is the spin of a spin wave? Phys. Lett. A 85, 375-377 (1981).

14. Castelnovo, C., Moessner, R. \& Sondhi, S. L. Magnetic monopoles in spin ice. Nature 451, 42-45 (2008).

15. Morris, D. J. P. et al. Dirac strings and magnetic monopoles in the spin ice $\mathrm{Dy}_{2} \mathrm{Ti}_{2} \mathrm{O}_{7}$. Science 326, 411-414 (2009).

16. Fennell, T. et al. Magnetic Coulomb phase in the spin ice $\mathrm{Ho}_{2} \mathrm{Ti}_{2} \mathrm{O}_{7}$. Science 326, 415-417 (2009).

17. Kadowaki, H. et al. Observation of magnetic monopoles in spin ice. J. Phys. Soc. Jpn 78, 103706 (2009).

18. Hao, Z. \& Tchernyshyov, O. Fermionic spin excitations in two- and three-dimensional antiferromagnets. Phys. Rev. Lett. 103, 187203 (2009).

19. Laughlin, R. B. Anomalous quantum Hall effect: An incompressible quantum fluid with fractionally charged excitations. Phys. Rev. Lett. 50, 1395-1398 (1983).

20. Wilczek, F. Quantum mechanics of fractional-spin particles. Phys. Rev. Lett. 49, 957-959 (1982).

21. Halperin, B. I. Statistics of quasiparticles and the hierarchy of fractional quantized Hall states. Phys. Rev. Lett. 52, 1583-1586 (1984).

22. Kukushkin, I. V. et al. Dispersion of the excitations of fractional quantum Hall states. Science 324, 1044-1047 (2009).

23. Su, W-P. \& Schrieffer, J. R. Fractionally charged excitations in charge-density-wave systems with commensurability 3. Phys. Rev. Lett. 46, 738-741 (1981).

24. Heeger, A. J., Kivelson, S., Schrieffer, J. R. \& Su, W-P. Solitons in conducting polymers. Rev. Mod. Phys. 60, 781-850 (1988).

25. Greiter, M. \& Thomale, R. Non-Abelian statistics in a quantum antiferromagnet. Phys. Rev. Lett. 102, 207203 (2009).

26. Yao, H., Zhang, S-C. \& Kivelson, S. A. Algebraic spin liquid in an exactly solvable spin model. Phys. Rev. Lett. 102, 217202 (2009).

27. Karbach, M. et al. Two-spinon dynamic structure factor of the one-dimensional $S=1 / 2$ Heisenberg antiferromagnet. Phys. Rev. B 55, 12510-12517 (1997).

28. Caux, J-S. \& Hagemans, R. The four-spinon dynamical structure factor of the Heisenberg chain. J. Stat. Mech.: Theory Exp. P12013 (2006).

29. Heilmann, I. U. et al. Neutron study of the line-shape and field dependence of magnetic excitations in $\mathrm{CuCl}_{2} \cdot 2 \mathrm{~N}\left(\mathrm{C}_{5} \mathrm{D}_{5}\right)$. Phys. Rev. B 18, 3530-3536 (1978).

30. Nagler, S. E. et al. Spin dynamics in the quantum antiferromagnetic chain compound $\mathrm{KCuF}_{3}$. Phys. Rev. B 44, 12361-12368 (1991).

31. Tennant, D. A., Perring, T. G., Cowley, R. A. \& Nagler, S. E. Unbound spinons in the $S=1 / 2$ antiferromagnetic chain $\mathrm{KCuF}_{3}$. Phys. Rev. Lett. 70, 4003-4006 (1993).

32. Arai, M. et al. Quantum spin excitations in the spin-Peierls system $\mathrm{CuGeO}_{3}$. Phys. Rev. Lett. 77, 3649-3652 (1996).

33. Dender, D. C. et al. Magnetic properties of a quasi-one-dimensional $S=1 / 2$ antiferromagnet: Copper benzoate. Phys. Rev. B 53, 2583-2589 (1996).

34. Zheludev, A. et al. Energy separation of single-particle and continuum states in an $S=1 / 2$ weakly coupled chains antiferromagnet. Phys. Rev. Lett. 85, 4799-4802 (2000).

35. Coldea, R., Tennant, D. A., Tsvelik, A. M. \& Tylczynski, Z. Experimental realization of a 2D fractional quantum spin liquid. Phys. Rev. Lett. 86, 1335-1338 (2001).

36. Stone, M. B. et al. Extended quantum critical phase in a magnetized spin-1/2 antiferromagnetic chain. Phys. Rev. Lett. 91, 037205 (2003).

37. Zaliznyak, I. A. et al. Spinons in the strongly correlated copper oxide chains in $\mathrm{SrCuO}_{2}$. Phys. Rev. Lett. 93, 087202 (2004).

38. Lake, B., Tennant, D. A., Frost, C. D. \& Nagler, S. E. Quantum criticality and universal scaling of a quantum antiferromagnet. Nature Mater. 4 329-334 (2005).

39. Hong, T. et al. Synthesis and structural characterization of 2Dioxane $\cdot 2 \mathrm{H}_{2} \mathrm{O} \cdot \mathrm{CuCl}_{2}$ : Metal-organic compound with Heisenberg antiferromagnetic $S=1 / 2$ chains. Phys. Rev. B 80, 132404 (2009).

40. Thielemann, B. et al. Direct observation of magnon fractionalization in the quantum spin ladder. Phys. Rev. Lett. 102, 107204 (2009).

41. Walters, A. C. et al. Effect of covalent bonding on magnetism and the missing neutron intensity in copper oxide compounds. Nature Phys. 5, 867-872 (2009).

42. Lake, B. et al. Confinement of fractional quantum number particles in a condensed-matter system. Nature Phys. 6, 50-55 (2010).

43. Enderle, M. et al. Two-spinon and four-spinon continuum in a frustrated ferromagnetic spin-1/2 chain. Phys. Rev. Lett. 104, 237207 (2010). 
44. Coldea, R. et al. Direct measurement of the spin Hamiltonian and observation of condensation of magnons in the $2 \mathrm{D}$ frustrated quantum magnet $\mathrm{Cs}_{2} \mathrm{CuCl}_{4}$. Phys. Rev. Lett. 88, 137203 (2002).

45. Des Cloizeaux, J. \& Pearson, J. J. Spin-wave spectrum of the antiferromagnetic linear chain. Phys. Rev. 128, 2131-2135 (1962).

46. Müller, G., Thomas, H., Beck, H. \& Bonner, J. C. Quantum spin dynamics of the antiferromagnetic linear chain in zero and nonzero magnetic field. Phys. Rev. B 24, 1429-1467 (1981).

47. Van Tol, M. W. \& Poulis, N. J. A high-field phase transition in the linear chain compound $\mathrm{CuSO}_{4} \cdot 5 \mathrm{D}_{2} \mathrm{O}$. Physica 69, 341-353 (1973).

48. Jimbo, M. \& Miwa, T. Algebraic Analysis of Solvable Lattice Models Vol. 85 (CBMS Regional Conf. Ser. in Math., Amer. Math. Soc., 1995).

\section{Acknowledgements}

We acknowledge useful discussions with C. Broholm, B. Dalla Piazza, B. Fåk, B. Lake, C. Rüegg and A. Tennant. The work of M.M. was supported in part by US Department of Energy (DOE), Office of Basic Energy Sciences, Division of Materials Sciences and Engineering under award DE-FG02-08ER46544. M.E. acknowledges suppor from the Deutsche Bundesministerium für Bildung, Wissenschaft, Forschung und
Technologie (BMBF), project 03KN5SAA. H.M.R. acknowledges support from the Swiss National Science Foundation (SNF) and the European Research Council (ERC). J-S.C. acknowledges support from the Foundation for Fundamental Research on Matter (FOM) and the Netherlands Organisation for Scientific Research (NWO).

\section{Author contributions}

M.E. and H.M.R. performed the experiment with the help of A.S. on a crystal synthesized by A.K. Data treatment and fits were carried out by M.M., M.E. and H.M.R.; exact theoretical calculations were performed by J-S.C. The physical pictures for multi-spinon excitations were developed through various discussions between J-S.C and M.M., M.E. and H.M.R. M.E., M.M., J-S.C. and H.M.R. wrote the manuscript.

\section{Additional information}

Supplementary information is available in the online version of the paper. Reprints and permissions information is available online at www.nature.com/reprints. Correspondence and requests for materials should be addressed to M.M

\section{Competing financial interests}

The authors declare no competing financial interests. 